\title{
Quiste teratoide congénito en piso de boca. Presentación de un caso y revisión de la literatura
}

\author{
Congenital teratoid cyst in the floor of the mouth. \\ Case report and literature review
}

\begin{abstract}
Alejandro Alonso Moctezuma,* Fabiola Salgado Chavarría, Eric Zeta Castañeda, $\$$ Leonor Robles Calzada, ${ }^{\S}$ Javier Portilla Robertson," Adriana Molotla Fragoso"
\end{abstract}

\section{RESUMEN}

Los quistes dermoides son lesiones congénitas benignas localmente invasivas de lento crecimiento, derivadas de células pluripotenciales. Se clasifican de acuerdo a los derivados embrionarios que contienen, se agrupan en dermoides, epidermoides y teratoides. Presentamos el caso clínico de un paciente de 12 años de edad con aumento de volumen sublingual con diagnóstico clínico de quiste dermoide. Se realizó resección quirúrgica mediante enucleación. El estudio histopatológico reportó quiste teratoide. Se dio seguimiento clínico durante 15 meses sin evidencia de recidiva.

Palabras clave: Quiste teratoide, quiste dermoide, enucleación, patología.

\section{ABSTRACT}

Dermoid cysts are congenital locally invasive benign lesions of slow growth, derived from pluripotent cells. They are classified according to the embryonic derivatives they contain; grouping them in dermoids, epidermoids and teratoids. Here we present a clinical case of a twelveyear-old male patient with sublingual growth diagnosed as a dermoid cyst. Surgical resection by enucleation was performed. The histopathological study reported a teratoid cyst. Clinical follow up from the next 15 months didn't showed recurrence.

Keywords: Teratoid cyst, dermoid cyst, enucleation, pathology.

* Médico Adscrito de la Especialidad en Cirugía Oral y Maxilofacial, División de Estudios de Postgrado e Investigación.

₹ Adscrita Especialidad en Cirugía Maxilofacial.

$\S$ Residente del Departamento de Patología Bucal y Maxilofacial.

" Coordinador del Departamento de Patología Bucal y Maxilofacial.

"Adscrita al Departamento de Patología Bucal y Maxilofacial.

División de Estudios de Postgrado e Investigación. Universidad Nacional Autónoma de México.

Correspondencia:

Alejandro Alonso Moctezuma

Departamento de Cirugía Oral y Maxilofacial, División de Estudios de Postgrado e Investigación.

Universidad Nacional Autónoma de México,

Investigación Científica Núm. 1853, CU, 04360,

Alcaldía Coyoacán, Ciudad de México, CDMX

Tel: 5529144347

E-mail: cmfalonsomoctezuma@ hotmail.com 


\section{INTRODUCCIÓN}

Los quistes dermoides son neoplasias de comportamiento benigno que pueden presentarse desde el nacimiento, de crecimiento lento, pero localmente invasivas derivadas de células con la habilidad de diferenciarse de cualquiera de los tejidos procedentes de las tres capas germinales. En 1937, New y Erich publicaron una serie de 1,495 pacientes con este diagnóstico, de los cuales, $23.3 \%$ se localizaban en el piso de boca. Meyer realizó una clasificación de acuerdo a los derivados embrionarios que contienen, los agrupó en: 1) epidermoides, quistes revestidos de epitelio, pero sin estructuras anexas, 2) dermoides, quistes con anexos dérmicos, 3) teratoides, quistes revestidos de epitelio que contienen elementos epiteliales y no epiteliales como hueso, músculo, tejido respiratorio o gastrointestinal y por último 4) teratomas verdaderos. ${ }^{1-3}$

La etiología de estos quistes se atribuye al atrapamiento de detritus epitelial en la línea media durante la fusión del primer y segundo arco faríngeo durante la quinta semana de desarrollo embrionario. Su incidencia oscila entre 0.05 y $2 \%$, sin predilección por sexo, $7 \%$ se presenta en la cabeza o cuello; de éstos, $23 \%$ se localizan en la cavidad oral y $0.01 \%$ en el piso de la boca. ${ }^{4}$ En esta región se observan principalmente en el triángulo submentoniano en relación con el músculo milohioideo o en el piso de la boca por encima de este mismo músculo.

Las manifestaciones clínicas son diversas, dependen de la localización y de su tamaño. Pueden ser asintomáticos o mostrar sintomatología, cuando se presentan en piso de boca se asocian con disfagia, dificultad para el habla o la respiración. 1,3,4

La tomografía computarizada contrastada suele mostrar una lesión quística que envuelve el espacio submental y el piso de la boca. ${ }^{2-4}$

El tratamiento de elección es la resección quirúrgica, que consiste en la enucleación; el pronóstico es bueno si se retira por completo la lesión, la malignización es extremadamente rara. ${ }^{3-5}$

Reportamos el caso clínico de un quiste teratoide congénito en un paciente de 12 años; esta variante del quiste dermoide es infrecuente, por lo que realizamos una revisión de la literatura.

\section{REPORTE DEL CASO}

Paciente masculino de 12 años de edad acude a la clínica de Medicina Bucal de la División de Estu- dios de Postgrado e Investigación de la Universidad Nacional Autónoma de México, sin antecedentes de importancia para su afección. Inicia padecimiento actual al nacer, presentando un aumento de volumen sublingual de crecimiento lento, pero progresivo, motivo por el que acudió con diversos facultativos sin recibir un diagnóstico y/o tratamiento definitivo.

A la exploración clínica se observó un aumento de volumen de $4 \times 4 \mathrm{~cm}$ localizado en la línea media del piso de la boca, con base amplia que desplazaba la lengua hacia arriba y atrás, proyectándose en la región submentoniana, sin cambios en la mucosa superficial, con signo en reloj de arena. El paciente presentaba eupnea, sin disnea, dolor, disfagia y desarrollo normal del lenguaje (Figura 1).

En la tomografía contrastada para tejidos blandos se observa área hipodensa a tejidos blandos de 4.5 $\mathrm{cm}$ en su diámetro mayor, de aspecto quístico con contenido isodenso a tejidos blandos (Figura 2).

Se realizó punción exploratoria obteniéndose $0.5 \mathrm{~cm}^{3}$ de contenido semilíquido de coloración amarillenta; en el análisis histológico del extendido se observaron queratinocitos y abundante queratina sobre un fondo fibrilar. Con base en los hallazgos clínicos y de imagen se estableció el diagnóstico clínico presuntivo de quiste dermoide. Posterior a la punción exploradora detectamos un aumento de tamaño del quiste, con presencia de dolor a la deglución, se llevó a cabo la resección quirúrgica bajo anestesia general, con abordaje transoral por medio de una incisión en la línea media de piso de boca conservando la integridad de los conductos de Wharton. Durante la disección se observó una firme adherencia de las fibras musculares a la cápsula quística y perforación del músculo milohiodeo; se resecó por completo la lesión quística para finalmente cerrar por planos con poliglactina 910 $4 / 0\left(\right.$ Vicry $\left.^{\circledR}\right)$. No se presentaron complicaciones (Figura 3).

La pieza quirúrgica fue fijada en formol amortiguado al $10 \%$ durante 24 horas. Macroscópicamente se trató de espécimen de tejido blando color café con áreas amarillas, que midió $5.0 \times 3.0 \times 2.5 \mathrm{~cm}$ de forma irregular, consistencia firme, superficie lisa con áreas anfractuosas y múltiples adherencias color marrón (Figura 3). Al corte se aprecia quístico, con contenido semilíquido color amarillo con abundantes estructuras esféricas blanco-amarillentas. Se incluyeron muestras representativas, las cuales fueron procesadas, cortadas a 5 micras y teñidas con hematoxilina y eosina. 
Figura 1:

(A) Imagen extraoral, se observa aumento de volumen en región submentoniana. (B) Intraoralmente se observa en línea media de piso de boca.

Figura 2:

(A) Tomografía computarizada contrastada en un corte axial y (B) corte sagital, se observa área

hipodensa a tejidos blandos bien delimitada, que desplaza la lengua hacia superior y posterior en contacto con el hueso hioides.
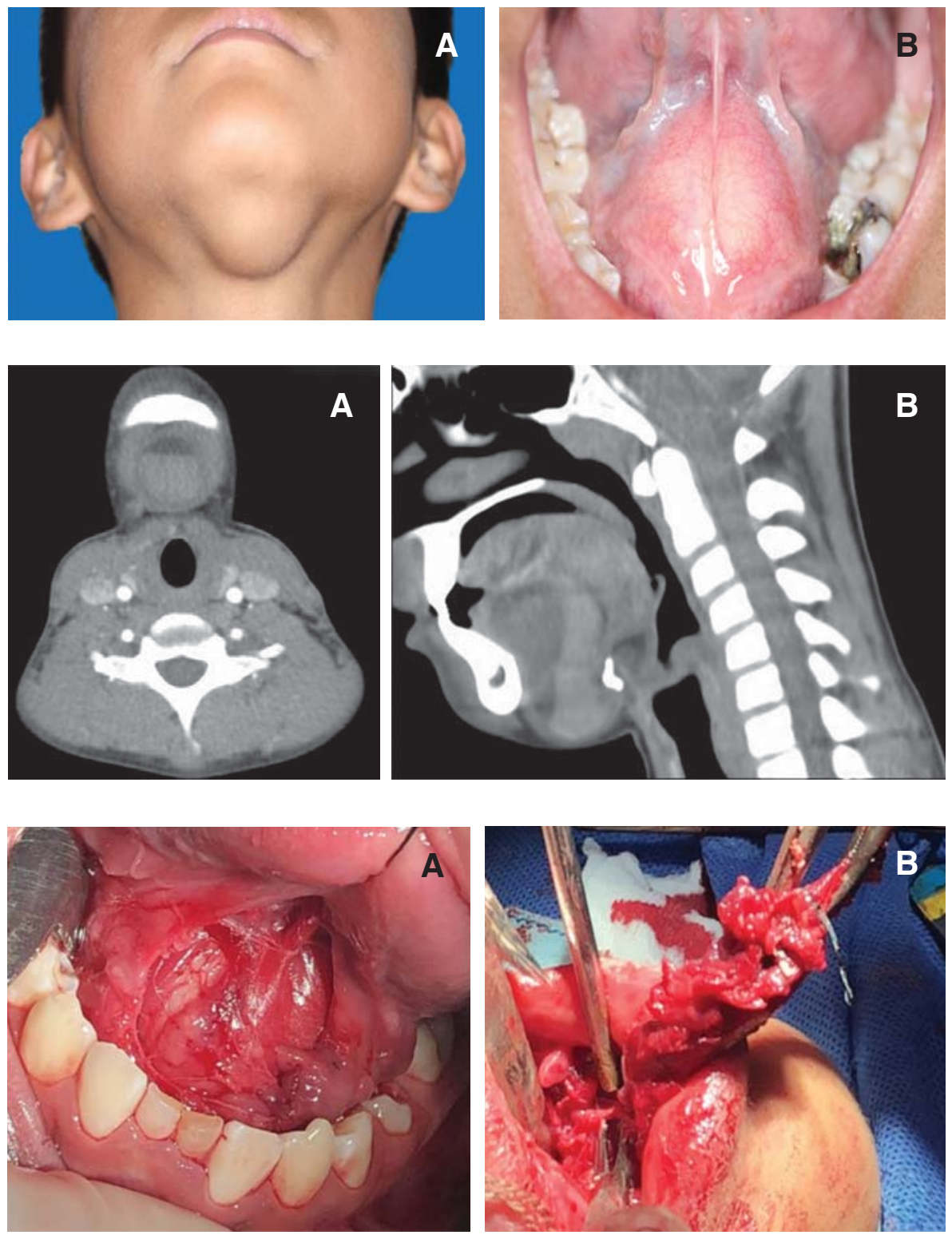

Figura 3:

(A) Incisión en la línea media de piso de boca, (B) disección de la lesión quística, (C) sutura por planos con poliglactina 910

(Vicryl ${ }^{\circledR}$ ) 4/0. Postquirúrgico inmediato, (D) espécimen fijado en formol al $10 \%$, que mide $5.0 x$

$3.0 \times 2.5 \mathrm{~cm}$, de forma irregular, color café, superficie lisa, con áreas anfractuosas.

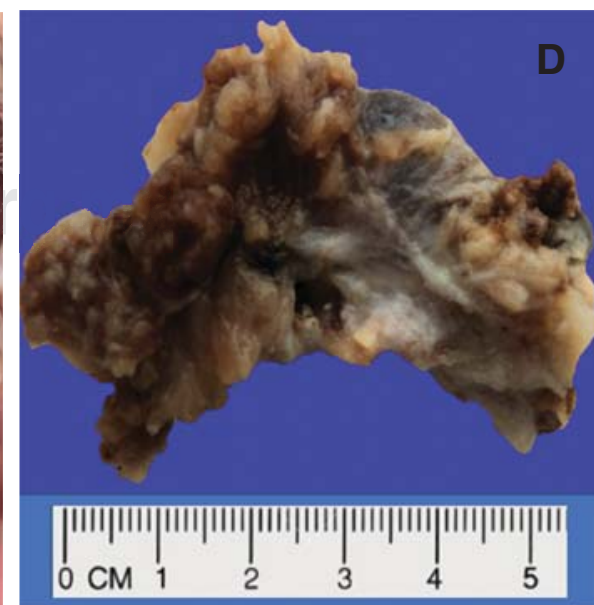

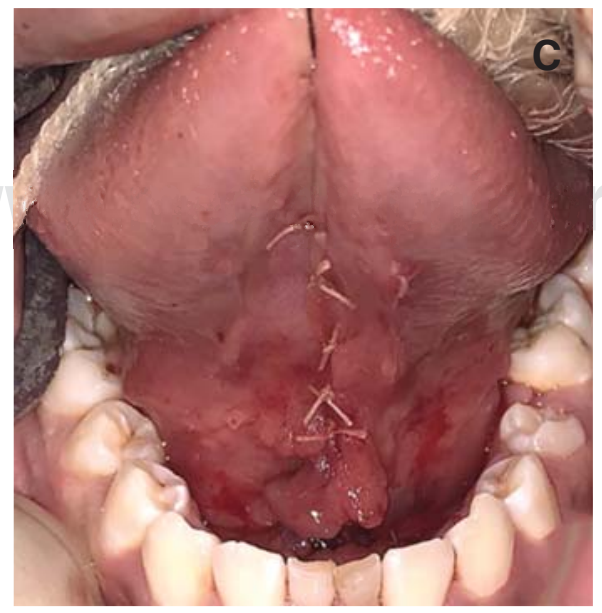


En los cortes histológicos examinados se observó una cavidad quística revestida por epitelio plano estratificado queratinizado de espesor variable; hacia la luz fue evidente la presencia de queratina con disposición en lámina. La cápsula de tejido conjuntivo fibroso denso contiene anexos cutáneos, glándulas sebáceas, folículos pilosos, también filetes de tejido nervioso de diferentes tamaños, haces de tejido muscular estriado esquelético y liso, abundantes vasos de diferentes calibres, algunos con estasis e infiltrado inflamatorio crónico linfoplasmocitario.
Hacia la periferia se observaron abundantes haces de tejido muscular estriado esquelético, tejido adiposo maduro y extravasación eritrocitaria. Con estos hallazgos se emitió el diagnóstico de quiste teratoide (Figura 4).

Se dio seguimiento clínico durante 15 meses sin evidencia de recidiva (Figura 5).

\section{DISCUSIÓN}

En 2013 Gordon y colaboradores utilizaron el término de quistes de fusión de la línea media para refe-
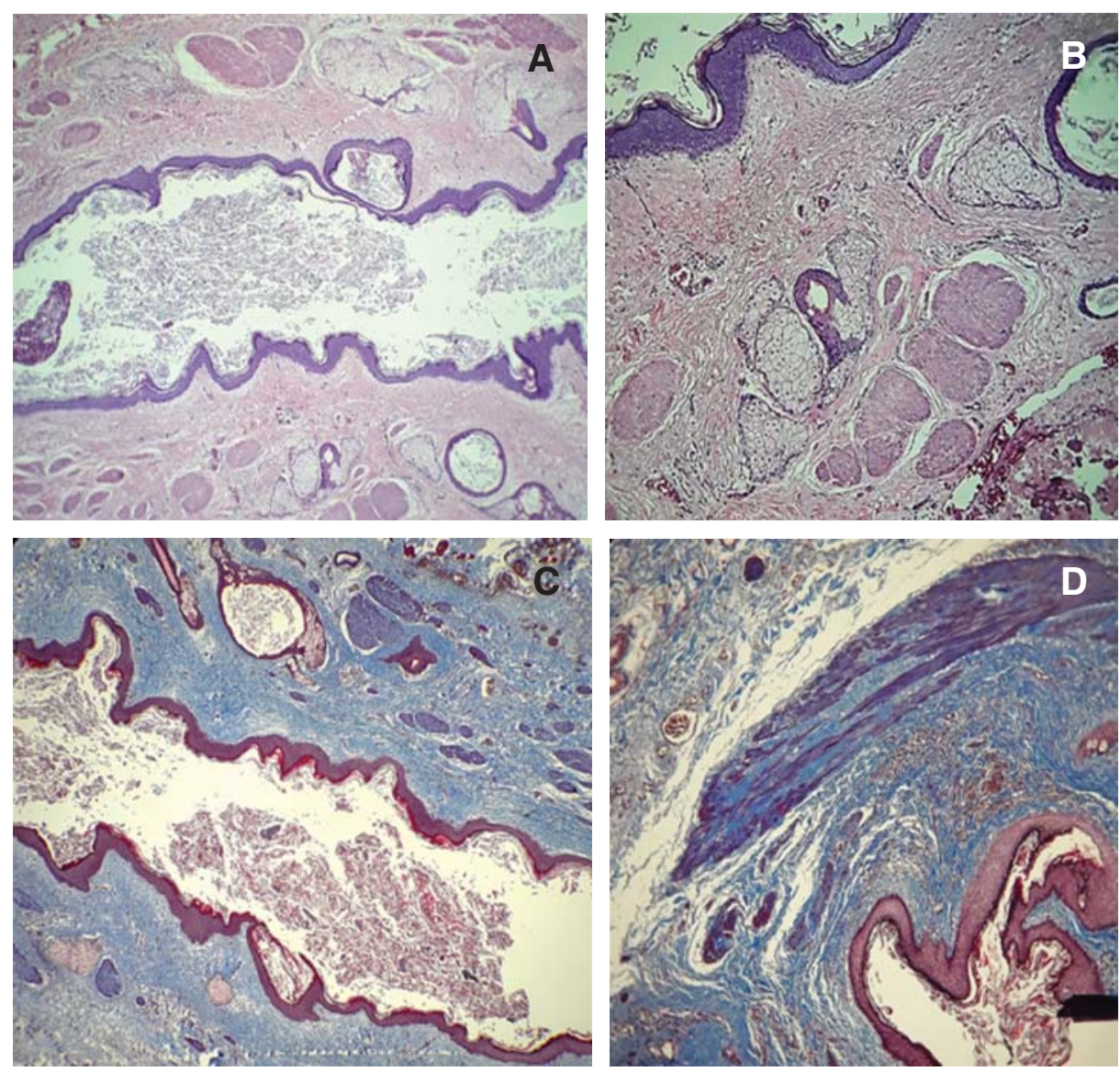

WN/VN/VA
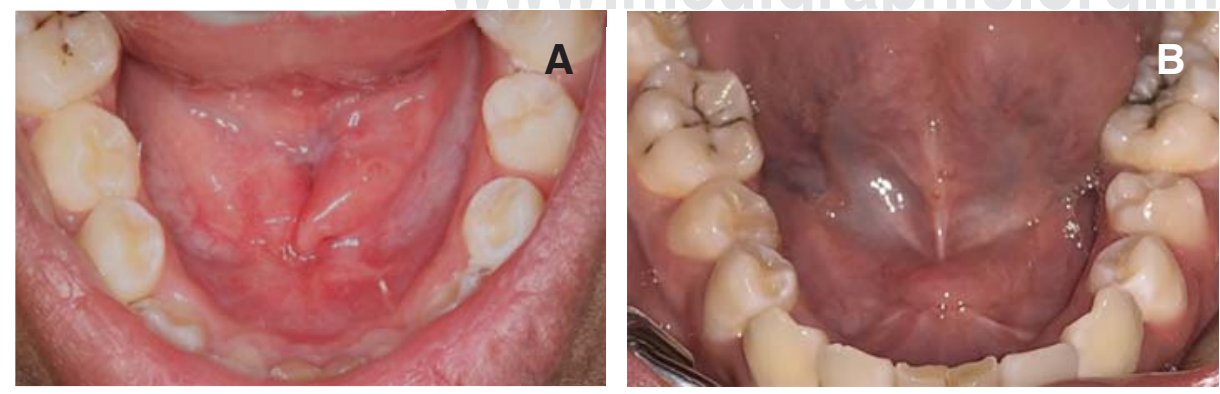

Figura 4:

(A) H\&E 40x Se observa una cavidad quística revestida por epitelio plano estratificado queratinizado, hacia la luz abundante queratina, (B) 100x en la cápsula de tejido conjuntivo fibroso denso, se aprecian anexos cutáneos, glándulas sebáceas, folículos pilosos, filetes de tejido nervioso y vasos de diferentes calibres, algunos con estasis, (C y D) Tricrómica de Masson a 40x y 100x. Son evidentes haces de tejido muscular estriado esquelético y músculo liso.

Figura 5:

(A y B) Fotografías intraorales de seguimiento (A, tres meses) observamos adecuada cicatrización y conductos salivales sin alteraciones. (B, 12 meses de seguimiento). 
rirse a los quistes dermoides; sin embargo, en la actualidad se conservan los términos propuestos en la clasificación de Meyer agrupados en quistes epidermoides, dermoides y teratoides. ${ }^{3,5,6}$

Los principales diagnósticos diferenciales incluyen: el quiste del conducto tirogloso, el cual se presenta clásicamente en la línea media cervical como un aumento de volumen asintomático, móvil y fluctuante desde el agujero ciego hasta la horquilla esternal, es muy raro encontrar esta lesión a nivel lingual. El quiste del segundo arco branquial por lo general muestra aumento de volumen laterocervical, no doloroso, y fijo a planos profundos. Los abscesos sublinguales y celulitis sublingual, ambos procesos infecciosos, que clínicamente pueden mostrar aumento de volumen, doloroso y fluctuante, la angina de Ludwig con frecuencia se asocia a un proceso infeccioso de origen dental. La ránula clínicamente presenta aumento de volumen sublingual unilateral y puede crecer hasta desplazar la lengua hacia atrás interfiriendo en la masticación y el habla, la mucosa superficial de la lesión con frecuencia es muy delgada adquiriendo un color blanco-azulado. El higroma quístico se trata de una anomalía congénita de los vasos linfáticos que impide el correcto drenaje de la linfa, es de consistencia blanda y fluctuante, con contenido líquido, su localización en orden de frecuencia es cervical, axilar e inguinal; sin embargo, esta patología congénita es diagnosticada al nacer. Estos diagnósticos diferenciales, en nuestro caso clínico fueron descartados con base en las características clínicas de imagen y el resultado de una biopsia por aspiración con aguja fina (BAAF) ${ }^{6-8}$

Los quistes dermoides se desarrollan con mayor frecuencia sobre el músculo milohioideo como tumores, que por lo regular están situados en la línea media del triángulo sublingual o piso de la boca, condicionan la elevación de la lengua y como consecuencia interfieren con la alimentación y/o el lenguaje. En nuestro caso clínico, el quiste perforó el músculo milohioideo; sin embargo, su crecimiento fue lento, por lo cual no mostró sintomatología. Los cambios que observamos en el quiste posterior a la punción exploratoria podrían explicarse debido a la respuesta inflamatoria, fibrosis y engrosamiento de la cápsula ocasionada por el trauma. ${ }^{7-10}$

El estudio de elección para el diagnóstico, localización y selección de la técnica quirúrgica es la tomografía computarizada contrastada para tejidos blandos; sin embargo, se pueden utilizar otros estudios no invasivos como la ultrasonografía de alta resolución y resonancia magnética. ${ }^{11-14}$

El abordaje transoral representa una técnica con adecuados resultados estéticos; generalmente los pacientes no presentan complicaciones y cuando la resección es completa, no se evidencia recurrencia. ${ }^{15-20}$

\section{CONCLUSIÓN}

En conclusión, debemos considerar que a pesar de ser el quiste teratoide una neoplasia benigna es importante identificar sus características clínicas, imagenológicas e histopatológicas. Nuestro paciente cursó con una evolución de 12 años, lo cual evidencia la necesidad de referir al especialista y la importancia de establecer diagnósticos oportunos, ya que esto tendrá repercusiones directas en la terapéutica de elección, evolución y en la calidad de vida del paciente.

En este caso se decidió, con base en los hallazgos clínicos y de acuerdo a la literatura, realizar un abordaje quirúrgico transoral, ya que dicha técnica es la que más beneficios brinda para el paciente. Se observó adecuada evolución con buenos resultados estéticos y funcionales.

\section{BIBLIOGRAFÍA}

1. Adel K, John KC, Jennifer RG, Takashi T. WHO Classification of head and neck tumors. 4th Ed. Lyon: WHO; 2017.

2. Jamal AM, Zhao Y. Teratoid cyat off loor of the mounth: a clinicopathologic study of 20 Chinesse patient. Int J Surg Pathol. 2009; 17 (2): 111-115.

3. Gordon PE, Faquin WC, Labey E, Kaban L. Floor-of-mouth dermoid cysts: report of 3 variants and a suggested change in terminology. J Oral Maxillofac Surg. 2013; 71: 1034-1041.

4. Liang $Y$, Yang Sh, Zhang Y. Retrospective analysis of ten cases of congenital sublingual teratoid cyst. Eur J Pediatri Dent. 2012; 13 (4): 333-336.

5. Bonilla JA, Szeremeta W, Yellon RF, Nazif MM. Teratoid cyst of the floor of the mouth. Int J Pediatr Otorhinolaryngol. 1996; 38: 71-75.

6. Pentenero M, Marino R, Familiari U, Gandolfo S. Choristoma involving the floor of the mouth and the anterior tongue: a case of teratoid cyst with gastric and respiratory epithelia. J Oral Maxillofac Surg. 2013; 71: 1706-1711.

7. Palaskar SJ, Garde J, Narang B. Teratoid cyst of the oral cavity: a rare entity. J Oral Maxillofac Pathol. 2014; 18 (3): 469-471.

8. $\mathrm{He} \mathrm{J}, \mathrm{He} \mathrm{Y,} \mathrm{Zhu} \mathrm{H,} \mathrm{Wang} \mathrm{Y,} \mathrm{Qiu} \mathrm{W.} \mathrm{Congenital} \mathrm{huge} \mathrm{submandibular}$ and neck teratoid cyst in newborn. J Craniofacl Surg. 2015; 26 (2): 565-567.

9. Tazi N, Mahdoufi R, Barhmi I, Mahtar M. Teratoid cyst of the tongue: a rare variant of dermoid cyst. Eur Ann Otorhinolaryngol Head Neck Dis. 2016; 133: 447-448.

10. Akao I, Nobukiyo S, Kobayashi T, Kikuchi H, Koizuka I. A case of large dermoid cyst in the floor of the mouth. Auria Nasus Larynx. 2003; 30: 137-139.

11. Tandon PN, Gupta DS. Epidermoid cyst in the floor of mouth with sub mental component. J Maxillofaac Oral Surg. 2014; 13 (1): 59-62. 
12. Kyriakidou E, Howe T, Veale B, Atkins S. Sublingual dermoid cyst: casa report and review of the literature. J Laryngol Otol. 2015; 129: 1036-1039.

13. Lin WH, Silver AL, Cunnane ME, Sadow PM, Kieff DA. Lateral dermoid cyst of the floor of mouth: unusual radiologic and pathologic findings. Auris Nasus Larynx. 2011; 38: 650-653.

14. Jain H, Sanjay S, Singh A. Giant sublingual dermoid cyst in floor of the mouth. J Maxillofac Oral Surg. 2012; 11 (2): 235-237.

15. Pan MB, Nakamura DD, Clark MD, Eisig DD. Intraoral dermoid cyst in an infant: a case report. J Oral Maxillofac Surg. 2011; 69: 1398-1402.

16. Bonet-Coloma C, Mínguez-Martínez I, Palma-Carrió C, OrtegaSánchez B, Peñarrocha-Diago MA, Mínguez-Sanz JM. Orofacial dermoid cysts in pediatric patients: a review of 8 cases. Med Oral Patol Oral Cir Bucal. 2011; 16 (2): 200-203.

17. Dilon JR, Avillo AJ, Nelson BL. Dermoid cyst of the floor of the mouth. Head Neck Pathol. 2015; 9: 376-378.

18. Marcal VE, Henrique BA, Ricci VL, Nogueir PA, Aburad CA, Almeida BG, Coelho BM. Unusual dermoid cyst in oral cavity. Hindawi. Case Rep Pathol. 2014: 1-3.

19. Edwards RM, Chapman TD, Horn DL, Paladin AM, lyer RS. Imaging of pediatric floor of mouth lesions. Pediatr Radiol. 2013; 45: 523-535.

20. Schwanke TW, Oomen KP, April MM, Ward VM. Floor of mouth masses in children: Proposal of a new algorithm. Int J Pediatr Otorhinolaryngol. 2013; 77: 1489-1494. 